\title{
On Implementing MPI-IO Portably and with High Performance*
}

\author{
Rajeev Thakur William Gropp Ewing Lusk \\ Mathematics and Computer Science Division \\ Argonne National Laboratory \\ Argonne, IL 60439, USA \\ \{thakur, gropp, lusk\} @mcs.anl.gov \\ Preprint ANL/MCS-P732-1098 \\ October 1998
}

\begin{abstract}
We discuss the issues involved in implementing MPI-IO portably on multiple machines and file systems and also achieving high performance. One way to implement MPI-IO portably is to implement it on top of the basic Unix I/O functions (open, lseek, read, write, and close), which are themselves portable. We argue that this approach has limitations in both functionality and performance. We instead advocate an implementation approach that combines a large portion of portable code and a small portion of code that is optimized separately for different machines and file systems. We have used such an approach to develop a high-performance, portable MPI-IO implementation, called ROMIO.

In addition to basic I/O functionality, we consider the issues of supporting other MPI-IO features, such as 64-bit file sizes, noncontiguous accesses, collective I/O, asynchronous I/O, consistency and atomicity semantics, user-supplied hints, shared file pointers, portable data representation, file preallocation, and some miscellaneous features. We describe how we implemented each of these features on various machines and file systems. The machines we consider are the HP Exemplar, IBM SP, Intel Paragon, NEC SX-4, SGI Origin2000, and networks of workstations; and the file systems we consider are HP HFS, IBM PIOFS, Intel PFS, NEC SFS, SGI XFS, NFS, and any general Unix file system (UFS).

We also present our thoughts on how a file system can be designed to better support MPI-IO. We provide a list of features desired from a file system that would help in implementing MPI-IO correctly and with high performance.
\end{abstract}

${ }^{*}$ This work was supported by the Mathematical, Information, and Computational Sciences Division subprogram of the Office of Computational and Technology Research, U.S. Department of Energy, under Contract W-31-109-Eng-38; and by the Scalable I/O Initiative, a multiagency project funded by the Defense Advanced Research Projects Agency (contract number DABT63-94-C-0049), the Department of Energy, the National Aeronautics and Space Administration, and the National Science Foundation. 


\section{Introduction}

Portable parallel programming has long been hampered by the lack of a standard, portable application programming interface (API) for parallel I/O. Most parallel file systems have a Unix-like API with variations that are nonportable. Furthermore, the Unix API is not an appropriate API for parallel I/O; it lacks some of the features necessary to express access patterns common in parallel programs, such as noncontiguous accesses and collective I/O, resulting in poor performance [34]. To overcome these limitations, the MPI Forum defined a new API for parallel I/O (which we call MPI-IO) as part of the MPI-2 standard [17]. MPI-IO is a comprehensive API with many features intended specifically for I/O parallelism, portability, and high performance. Implementations of MPI-IO, both portable and machine-specific, are beginning to appear $[6,12,22,23,33]$.

In this paper, we discuss the issues involved in implementing MPI-IO portably on multiple machines and file systems and also achieving high performance. We argue that if an implementation uses just the basic Unix I/O functions in order to achieve portability, it will have limitations in both functionality and performance. We describe an alternative approach, called ADIO, that achieves portability and performance by combining a large portion of portable code with a small portion of code that is optimized separately for different machines and file systems. We have used this approach in our portable MPI-IO implementation, ROMIO. ${ }^{1}$

In addition to implementing basic I/O functionality (open, close, read, write, seek), we consider the issues of supporting other MPI-IO features, such as 64-bit file sizes, noncontiguous accesses, collective I/O, asynchronous I/O, consistency and atomicity semantics, user-supplied hints, shared file pointers, portable data representation, file preallocation, and some miscellaneous features. We describe how we implemented each of these features on various machines and file systems. The machines we consider are the HP Exemplar, IBM SP, Intel Paragon, NEC SX-4, SGI Origin2000, and networks of workstations; and the file systems we consider are HP HFS, IBM PIOFS, Intel PFS, NEC SFS, SGI XFS, the Network File System (NFS), and any general Unix file system (UFS).

We also describe how a file system can be designed to better support MPI-IO. We provide a list of features desired from a file system that would help in implementing MPI-IO correctly and with high performance.

\section{Achieving Portability and Performance}

The basic Unix I/O functions (open, lseek, read, write, and close) [28] are supported without variation on all machines with a Unix-like operating system. One way to implement MPI-IO portably, therefore, is to implement MPI-IO functions on top of the basic Unix I/O functions. Since the Unix I/O functions are portable, such an MPI-IO implementation will be portable to many machines and file systems. This approach, however, has limitations in both functionality and performance, as explained below:

1. The basic Unix I/O functions are not sufficient to implement all of MPI-IO on all file systems for the following reasons:

- The basic Unix I/O functions are blocking functions. Many file systems provide a different set of (nonportable) functions for nonblocking I/O. ${ }^{2}$

- On many file systems, the basic Unix I/O functions work only on files of size less than 2 Gbytes. Different functions must be used for larger files, and these functions are also

\footnotetext{
${ }^{1}$ URL: http: //ww.mcs. anl.gov/romio

${ }^{2} \mathrm{It}$ is possible, however, to implement nonblocking I/O by spawning a thread that calls a blocking I/O function.
} 
nonportable. (We note that an MPI-IO implementation is not required to support large file sizes, but most high-quality implementations will.)

- Some file systems allow the user to control file-striping attributes with special, nonportable functions (e.g., IBM PIOFS and Intel PFS).

- Some file systems support additional features such as file preallocation (e.g., SGI XFS, Intel PFS, HP HFS) and a choice of atomic and nonatomic file-access modes (e.g., IBM PIOFS and Intel PFS). The corresponding functions are also nonportable.

Since all these features are available at the MPI-IO level, an MPI-IO implementation cannot support them if it uses only the basic Unix I/O functions.

2. Although the basic Unix I/O functions are supported on all file systems, they are often not the recommended functions (for performance) on all file systems. For example,

- On the Intel Paragon, the recommended functions are cread and cwrite.

- On SGI IRIX 6.5, the recommended functions are pread64 and pwrite64; on IRIX 6.4 and earlier, they are called pread and pwrite.

- On HP machines running the SPPUX operating system (and not HPUX), the recommended functions are pread64 and pwrite64.

3. When using the Network File System (NFS), it is not sufficient to call just the Unix read/write functions. Since NFS performs noncoherent client-side caching by default, file consistency is not guaranteed if multiple processes write to a common file [27]. Client-side caching must be disabled by locking the portion of the file being accessed, by using fontl. A lock and unlock are therefore needed across the read/write call.

4. Many research file systems provide their own APIs $[8,3,10,14,19]$. Implementing MPI-IO on top of Unix I/O functions will not be portable to these file systems.

An alternative is to implement MPI-IO on top of the POSIX I/O interface [11] instead of the basic Unix I/O functions. The POSIX interface is an international standard with greater functionality than basic Unix I/O. For example, POSIX supports asynchronous I/O and list-directed I/O. This approach, however, also has limitations. Although POSIX is a standard, it is not yet widely implemented. One, therefore, cannot assume that POSIX I/O functions will be available on all file systems. Furthermore, many vendors do not follow the POSIX standard strictly. They implement only parts of it, and even the implemented portion may not conform strictly to the standard (particularly in the case of asynchronous $\mathrm{I} / \mathrm{O})$. Some vendors provide a separate set of functions for 64-bit file sizes. POSIX also does not support some features that MPI-IO supports, for example, file preallocation and varying file-striping attributes. Nonstandard functions must be used on file systems that support these features. In all, implementing MPI-IO on top of POSIX I/O is not sufficient either.

We believe that the only way to implement MPI-IO portably with complete functionality and high performance is to have a mechanism that can utilize the special features and functions of each file system. We describe such an architecture, called ADIO, which we use in our MPI-IO implementation, ROMIO [33].

\subsection{Abstract-Device Interface for I/O}

A key component of ROMIO that enables such a portable MPI-IO implementation is an internal layer called ADIO [30]. ADIO, an abstract-device interface for $\mathrm{I} / \mathrm{O}$, is a mechanism specifically designed for 
implementing parallel-I/O APIs portably on multiple file systems. We developed ADIO before MPIIO became a standard, as a means to implement and experiment with various parallel-I/O APIs that existed at the time.

ADIO consists of a small set of basic functions for parallel I/O. Any parallel-I/O API can be implemented portably on top of ADIO, and ADIO itself is implemented separately on each different file system. ADIO thus separates the machine-dependent and machine-independent aspects involved in implementing an API. The ADIO implementation on a particular file system is optimized for that file system. We used ADIO to implement Intel's PFS API and subsets of IBM's PIOFS API and the original MPI-IO proposal [35] on PFS, PIOFS, Unix, and NFS file systems. By following such an approach, we achieved portability with very low overhead [30]. Now that MPI-IO has emerged as the standard, we use ADIO as a mechanism for implementing MPI-IO portably (see Figure 1), and this MPI-IO implementation is called ROMIO [33].

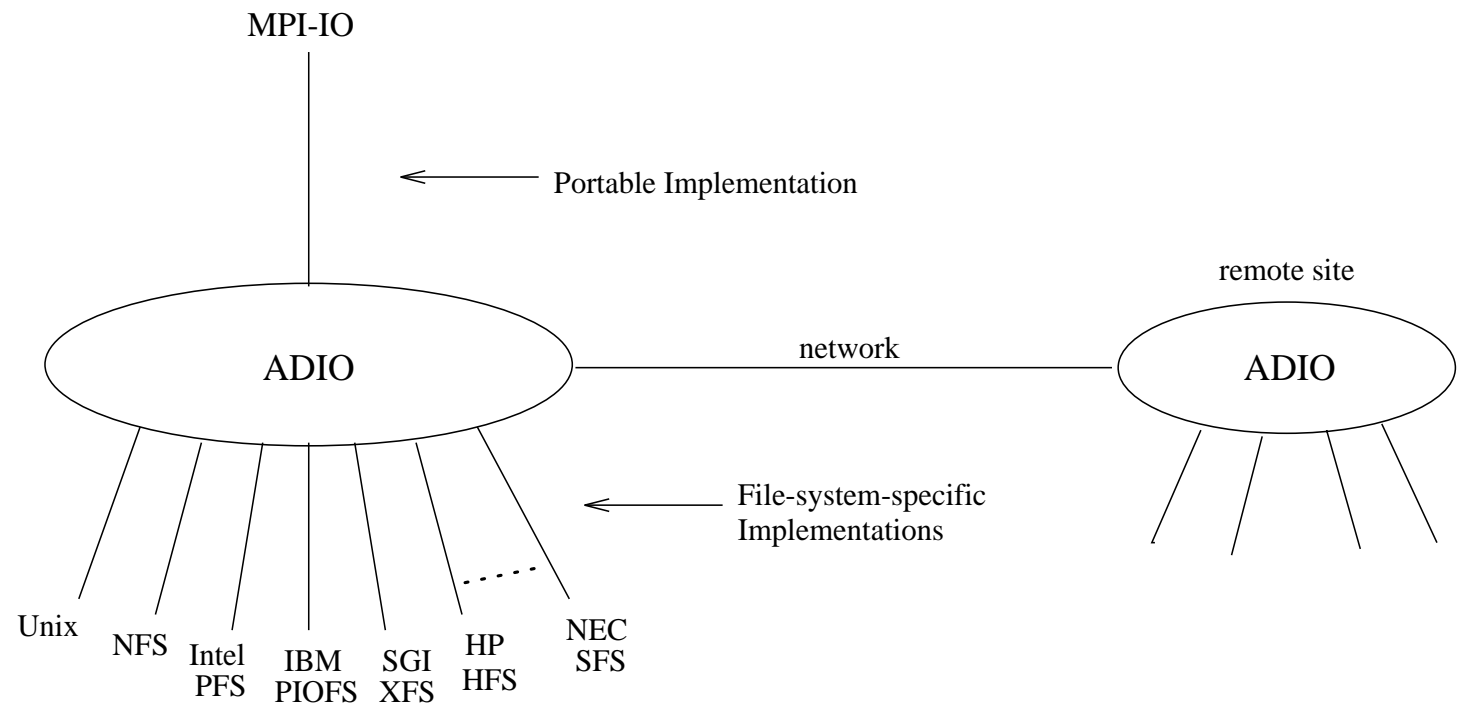

Figure 1: ROMIO architecture: MPI-IO is implemented portably on top of an abstract-device interface called ADIO, and ADIO is optimized separately for different file systems.

Another application of ADIO is for implementing remote I/O. An MPI-IO program running on one machine can access files from remote machines by developing an ADIO implementation that accesses data from an ADIO server running at a remote site. Such an implementation is described in [7] and also illustrated in Figure 1.

A similar abstract-device interface is used in MPICH [9] for implementing MPI portably.

\section{$3 \quad$ Implementing MPI-IO}

We describe how we implemented each feature of MPI-IO on various machines and file systems. The many variations among machines clearly demonstrate the need for an ADIO-like approach to implementing MPI-IO portably, where the variations are accounted for in the ADIO implementation.

\subsection{Basic File Access}

We first consider the basic file-access operations: open, close, read, write, and seek. We consider reads and writes in which data is contiguous in both memory and file; noncontiguous accesses are considered in Section 3.2. 


\subsubsection{Open}

MPI_File_open is a collective function. One of its arguments is an MPI communicator [18] that specifies the group of processes that will call this open function and any other MPI-IO collective function that the user may choose to use on the open file. Most file systems, other than Intel PFS, support only the regular Unix open and do not have collective open functions. On these file systems, ROMIO just calls the open function on each process. Intel PFS supports two open functions: regular Unix open and gopen. gopen is a "global open," recommended to be used when all processes in the application open a common file. It cannot be used when a subset of processes open the file; the function will hang if all processes do not call it.

MPI-IO also supports a few additional file-access modes that are not defined in Unix or POSIX.

\subsubsection{Close}

The close function on most file systems is identical to close in Unix or POSIX. MPIFile_close can be implemented in a straightforward manner on top of Unix close. If the file was opened with the mode MPI_MODE_DELETE_ON_CLOSE, the implementation must delete the file. Most file systems support the Unix function unlink for deleting a file.

\subsubsection{Large Files}

Most file systems distinguish between files of size less than 2 Gbytes and greater than or equal to 2 Gbytes. The reason is that file offsets and file sizes are usually represented by 4-byte integers in the regular I/O functions. The largest number that can be represented by a 4 -byte signed integer is $(2$ Gbytes -1$)$. With the regular file-system functions, therefore, it is not possible to access data from locations beyond 2 Gbytes. To overcome this problem, most file systems provide separate functions that use 8 -byte integers to represent file offsets.

In MPI-IO, file offsets are of type MPI_Offset, which is a data type defined by the MPI-IO implementation. The implementation is free to define it to be of any size; the standard does not mandate that the implementation support large files. In ROMIO, however, on those file systems that support large files (such as IBM PIOFS, HP HFS, NEC SFS, and SGI XFS), ROMIO treats all files as large files; that is, it defines MPI_Offset as an 8-byte integer and uses the corresponding file-system functions for large files (even though the file may be smaller than 2 Gbytes). On file systems that do not support large files, ROMIO also does not support large files and defines MPI_Offset as a 4-byte integer.

\subsubsection{Seek}

MPI-IO has two kinds of file pointers, individual and shared, and, correspondingly, two seek functions to move these file pointers. Most file systems (other than Intel PFS), however, support only individual file pointers. In Section 3.8 we describe how an MPI-IO implementation can implement shared file pointers on top of individual file pointers.

Most file systems support the Unix Iseek function. One some file systems we need to use a different function for large files: 1seek64 on SGI XFS, HP HFS, and NEC SFS; 1lseek on IBM PIOFS.

\subsubsection{Contiguous Reads and Writes}

Contiguous reads and writes in MPI-IO can be mapped directly onto the reads and writes of the underlying file system. The recommended functions for read and write, however, vary considerably among machines. ROMIO uses the following functions:

- _cread/_cwrite on Intel PFS. 
- pread64/pwrite64 on HP HFS if the operating system is SPPUX and read/write if it is HPUX.

- pread64/pwrite64 on SGI XFS if the operating system is IRIX 6.5. On IRIX 6.4 and earlier, the same functions are called pread/pwrite.

- $\mathrm{read} /$ write elsewhere.

The functions pread64/pwrite64 take the file offset as an argument; therefore, a separate 1seek64 is not required.

\subsection{Noncontiguous Accesses}

MPI-IO allows users to access noncontiguous data from a file into noncontiguous memory locations with a single I/O function call. The user can specify noncontiguous locations in the file by creating a file view with MPI's derived datatypes [17]. Noncontiguous locations in memory can be specified by using a derived datatype in the read/write call.

The ability of users to specify noncontiguous accesses in a single function call is very important, because noncontiguous accesses are very common in parallel applications [1, 4, 20, 25, 26, 31]. Most file systems, however, do not provide functions for noncontiguous I/O. The Unix functions readv/writev are widely supported, but they allow noncontiguity only in memory and not in the file. Noncontiguous memory accesses are not as commonly needed in parallel applications as noncontiguous file accesses. Furthermore, most file systems impose a limit of at most sixteen noncontiguous memory locations in a single readv/writev call.

Some file systems support the POSIX list-directed I/O function lio_listio, which allows users to submit multiple I/O requests at a time. This function also has limitations, because of the way it is defined. For one, it is tied too closely to nonblocking (asynchronous) I/O. The POSIX standard [11] says that each of the requests in the list will be submitted as a separate nonblocking request. It is known, however, that issuing too many nonblocking I/O requests at a time can lower performance. Furthermore, POSIX allows the requests in the list to be a mixture of reads and writes. All these specifications make it difficult for the POSIX implementation to optimize I/O for the entire list of requests, for example, by performing data sieving [32].

In the absence of proper support for noncontiguous I/O from the file system, one way to implement a noncontiguous MPI-IO request is to access each contiguous portion of the request separately by using the regular contiguous read/write functions of the file system. Such an implementation, however, results in a large number of small requests to the file system, and performance degrades drastically [32]. ROMIO instead performs an optimization called data sieving to access noncontiguous data with high performance. The basic idea in data sieving is to make large I/O requests to the file system and extract, in memory, the data that is really needed. Details of this optimization can be found in [32].

\section{$3.3 \quad$ Collective $\mathrm{I} / \mathrm{O}$}

MPI-IO provides collective-I/O functions, which must be called by all processes that together opened the file. ${ }^{3}$ This property enables the MPI-IO implementation (or file system) to analyze and merge the requests of different processes. In many cases, the merged request may be large and contiguous, although the individual requests of each process are noncontiguous. The merged request can therefore be serviced efficiently, and such optimization is broadly referred to as collective I/O. Collective I/O has been shown to be a very important optimization in parallel $\mathrm{I} / \mathrm{O}$ and can improve performance significantly $[5,13,24,29]$.

\footnotetext{
${ }^{3}$ An MPI communicator is used in the open call to specify the participating processes, and the communicator could represent any subset (or all) of the processes of the application.
} 
Since none of the file systems on which ROMIO is implemented perform collective I/O, ROMIO performs collective I/O on top of the file system. ROMIO's collective-I/O implementation is described in detail in [32], and it is a generalization of the two-phase method originally proposed in [5] and extended further in [29]. In two-phase I/O, as the name suggests, I/O is performed in two phases: a communication phase and an I/O phase. The communication phase uses interprocess communication to rearrange data into large chunks. In the I/O phase, processes perform parallel I/O in large chunks and therefore obtain high I/O performance.

For example, Figure 2 shows the performance of an astrophysics application template, DIST3D, when I/O is performed in three ways: using Unix-style independent I/O, data sieving, and collective I/O. This application accesses a three-dimensional distributed array of size $512 \times 512 \times 512$ from a file. Depending on the machine, data sieving performs either slightly better or much better than Unix-style independent I/O. Collective I/O always performs the best and results in I/O bandwidths of hundreds of megabytes/sec. For detailed performance results, see [32].
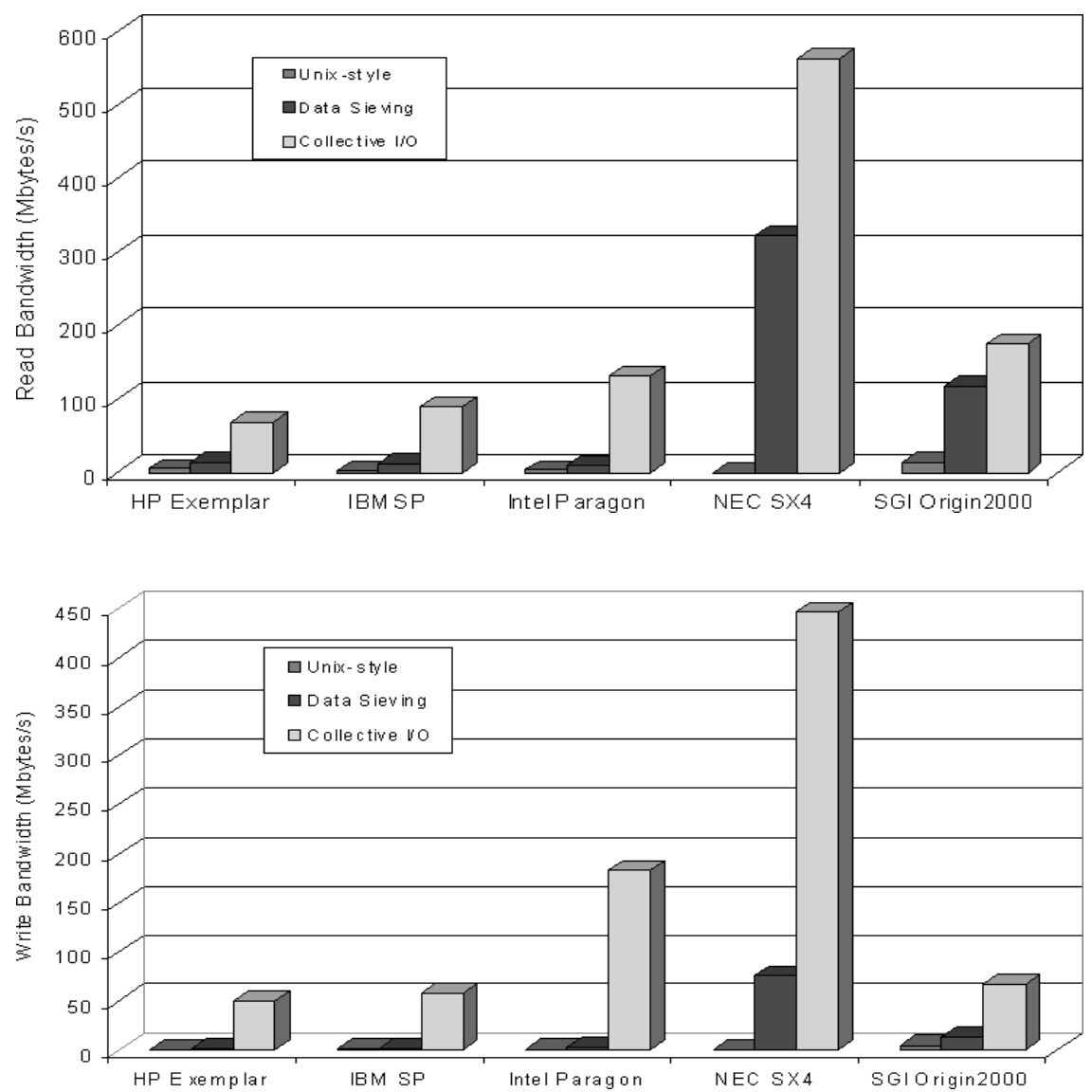

Figure 2: Performance of DIST3D using Unix-style independent I/O, data sieving, and collective I/O. The top figure shows read bandwidth, and the bottom figure shows write bandwidth.

\subsection{Nonblocking (Asynchronous) I/O}

Many file systems support nonblocking I/O, and one way to implement MPI-IO's nonblocking I/O functions is to use the nonblocking functions of the file system. Intel PFS supports nonstandard functions called iread and iwrite. Other vendors (SGI, IBM, DEC, Sun) support POSIX asynchronous I/O (aio) functions, but, in many cases, they do not follow the POSIX definition strictly. IBM supports 
nonblocking I/O on Unix and NFS file systems, but not on PIOFS. HP supports nonblocking I/O only on HPUX version 11.0 and higher, but not on SPPUX or earlier versions of HPUX. Nonblocking I/O functions are not yet available in Linux, FreeBSD, or on the NEC SX-4.

Another way to implement nonblocking I/O is by explicitly using threads that call blocking I/O functions. This approach, however, requires good thread support on the machine and a thread-safe MPI implementation, neither of which are common on parallel machines as yet.

ROMIO implements nonblocking I/O by using the nonblocking I/O functions of the file system where available. On machines and file systems that do not support nonblocking I/O, ROMIO just calls the corresponding blocking I/O functions.

\subsection{Consistency Semantics}

MPI-IO's consistency semantics (Section 9.6 of [17]) define the results users can expect with concurrent file accesses from multiple processes. MPI-IO's consistency semantics are actually weaker than the consistency semantics in Unix [28] or POSIX [11]. In Unix and POSIX, after a write function returns, the data is guaranteed to be visible to every other process in the system. MPI-IO guarantees that writes from one process are immediately visible only to those processes in the communicator that was used to open the file. Any other process can access the data only after both the writer and reader call MPI_File_sync.

MPI-IO's consistency semantics are therefore automatically guaranteed on file systems that support Unix consistency semantics. NFS, by default, does not [27]. To obtain Unix consistency semantics on NFS, ROMIO uses byte-range locking ( $f \mathrm{cntl}$ ) across the reads and writes in order to turn off the noncoherent client-side caching that NFS otherwise performs. Turning off client-side caching reduces performance considerably, but is, nonetheless, necessary for correctness. We believe that the other file systems on which ROMIO is implemented do support Unix consistency semantics correctly.

\subsection{Atomicity Semantics}

Atomicity semantics define the results when multiple processes issue concurrent requests to overlapping regions in the file, and one or more of those requests are write requests. MPI-IO supports two atomicity modes. The default mode is nonatomic, in which the results of such concurrent requests are undefined. The user can change the mode to atomic, in which case the overlapping region will contain data from any one process only.

The atomic mode is the only mode supported in Unix and POSIX. On file systems that support Unix atomicity semantics correctly, the atomic mode is therefore implemented by default, at least for contiguous MPI-IO requests. If the MPI-IO request is noncontiguous in the file, however, and the implementation writes it by making more than one write function call, then atomicity is not guaranteed for the entire noncontiguous MPI-IO request. To guarantee atomicity in such cases (when the user has set atomic mode and the request is noncontiguous), ROMIO locks the range of bytes being accessed in the file and then performs the necessary I/O.

On file systems that support only the atomic mode, the nonatomic mode is also implemented by default, since it has weaker semantics than the atomic mode. Some file systems, such as IBM PIOFS and Intel PFS, support both modes, because it is possible for the file system to deliver higher performance when the access mode is nonatomic. On PIOFS, the default mode is nonatomic (called NORMAL); the user can change the access mode to atomic (called CAUTIOUS) with the function piofsioctl. On PFS, the default mode is atomic (called M_UNIX); nonatomic mode (called M_ASYNC) can be selected by using either the function gopen or setiomode. Both gopen and setiomode, however, are "global" functions: all processes in the application must call them. In MPI-IO, a user can create a communicator 
containing a subset of all processes and open the file with this communicator. In such cases, the MPI-IO implementation cannot use the nonatomic mode on PFS.

\subsection{Hints}

MPI-IO provides a mechanism for the user to pass hints to the implementation. Hints, such as accesspattern information, can help the implementation optimize file access [2, 21]. Hints do not change the semantics of the MPI-IO interface; an implementation may choose to ignore all hints, and the program would still be functionally correct. MPI-IO has some predefined hints for specifying file-striping parameters, access patterns, and so on. An implementation is free to define additional hints.

ROMIO supports some predefined hints and some additional hints. The predefined hints supported are the file-striping parameters (number of disks and striping unit) and the buffer size and number of processes to use for collective I/O. Additional hints supported by ROMIO are the disk number from which to begin striping the file, buffer sizes for data sieving, and, on Intel PFS only, a hint to turn on server buffering. ROMIO uses the file-striping hints only on the two file systems that allow the striping parameters to be varied, namely, Intel PFS and IBM PIOFS; they are ignored on other file systems. On PFS, ROMIO uses the fontl function to vary file-striping parameters. On PIOFS, the function is piofsioctl.

MPI-IO also allows users to query the current value of a hint. With this feature, users can, for example, determine the default file-striping parameters or the buffer sizes ROMIO uses for data sieving and collective I/O.

\subsection{Shared File Pointers}

Most file systems, other than Intel PFS, do not support shared file pointers. On such file systems, the MPI-IO implementation must implement shared file pointers above the file system. Doing so requires some mechanism for maintaining the value of the shared file pointer for each file and for processes to access and atomically update this value. One way is to store the value of the shared file pointer in a file and have processes update the value atomically by using file locks. Another way is to have one process or thread maintain the value, and other processes can access the value from this process or thread. ROMIO does not use the latter approach because it requires that the MPI implementation support dynamic processes, or one-sided communication, or multiple threads, and none of these features are commonly supported by MPI implementations as yet.

ROMIO stores the value of the shared file pointer in a file in the same directory as the data file being accessed. When a process needs to access data using the shared file pointer, it locks the file containing the shared-file-pointer value, reads the value, increments it by the amount of data to be read or written, writes the new value back, releases the lock, and then performs the read or write of actual data. The shared-file-pointer file is created when the shared file pointer is first used in the program and is deleted when the user closes the data file.

\subsection{Portable Data Representation}

MPI-IO supports multiple data-storage representations: native, internal, externa132, and also userdefined representations. native means that data is stored in the file as it is in memory; no data conversion is performed. internal is an implementation-defined representation that may provide some degree of file portability. external32 is a specific, portable data representation defined in MPI-IO. A file written in external32 format on one machine is guaranteed to be readable on any machine with any MPI-IO implementation. MPI-IO also provides a mechanism for users to define a new data 
representation by providing data-conversion functions, which MPI-IO uses to convert data from file format to memory format and vice versa.

The native representation is implemented by default, and an implementation can use external32 as its internal representation. One way to implement external32 is to convert each datatype explicitly from/to the external32 representation, which may require byte swapping, truncation, or padding, depending on the machine. Another way to implement external32 is via the data-conversion functions: The implementation can provide the data-conversion functions to translate from external32 to native representation and vice versa, and the implementation itself will use these functions to support external32.

ROMIO currently supports only the native representation. We plan to implement external32 via the data-conversion functions, mainly because this approach is modular, easily extensible to new platforms, and also serves as a template that users can use to define other data representations.

\subsection{File Preallocation}

Only a few file systems provide a function to preallocate disk space for a file. Intel PFS has a function called lsize, on SGI XFS one can preallocate space via fcntl, and HP HFS has functions prealloc and prealloc64. On other file systems that do not support file preallocation, the MPI-IO implementation must allocate space by actually writing data to the file (which is expensive).

\subsection{Miscellaneous Issues}

Here we consider some miscellaneous issues in implementing MPI-IO.

\subsubsection{Library versus Client-Server Implementation}

An MPI-IO implementer is faced with the choice of implementing it as a library or as a client-server implementation. We believe that if the underlying file system supports high-performance access from multiple processes to a common file, a library approach is sufficient. Any further optimizations needed, such as data sieving and collective I/O, can be implemented within the library. Such is the case on parallel machines like the IBM SP, Intel Paragon, SGI Origin2000, HP Exemplar, and NEC SX-4.

A client-server approach is needed if no common file system exists for all processes to access, for example, when the processes run on clusters of independent machines, each with their own local file system. In such a case, the MPI-IO implementation would need to have servers that implement a virtual shared file system on top of the individual file systems on these machines. Another example is when MPI-IO is used to access files from remote machines, as described in [7].

\subsubsection{Operating with Multiple MPI-1 Implementations}

MPI-IO can be implemented in a way that it can operate with any MPI-1 implementation that also has a few functions defined in the MPI-2 external-interfaces chapter. These functions allow the MPI-IO implementation to access a few internal data structures of the MPI implementation.

ROMIO is designed to operate with multiple MPI implementations. It currently works with MPICH, HP MPI, and SGI MPI, because these implementations provide the external-interface functions ROMIO needs.

\subsubsection{Automatic Detection of File-System Type}

ROMIO allows users to access files on multiple file systems in the same program; therefore, it needs to know the type of file system on which a given file resides. The first version of ROMIO (1.0.0) required 
users to prefix the filename with a string (like nfs:) indicating the type of file system. This requirement can be inconvenient to users and can make programs nonportable. In the next version of ROMIO, we therefore eliminated this requirement. ROMIO can now determine the file-system type on its own by using functions that are available for this purpose on most file systems. ${ }^{4}$ On most file systems, the function is statvfs, on some it is statfs, on Intel PFS it is statpfs, and on the NEC SX-4 it is stat.

\subsubsection{Automatic Configure and Build}

Many parts of the ROMIO source code are conditionally compiled, depending on the features of the environment (machine, file system, MPI implementation). These features are detected automatically by using GNU's autoconf utility. We distribute ROMIO in the form of source code, and users can build it on any machine by simply doing

$\%$ configure

$\%$ make

We learnt early on to have the configure script look for features of a particular environment and not for things like version numbers of the underlying operating system and other software. By following this approach, we are able to adapt easily to constantly changing version numbers and features. Users are also able to build ROMIO easily on new environments where we, the developers, had never before built or tested ROMIO.

\section{Implications for File-System Design}

File-system designers may want to know how they could design their file system to better support MPIIO. We provide a list of features desired from a file system that would help in implementing MPI-IO correctly and with high performance.

1. High-Performance Parallel File Access. The file system must be designed to support highperformance access from multiple processes to a common file. This implies that concurrent requests (particularly writes) must not be serialized within the file system.

2. Data-Consistency Semantics. The data-consistency semantics in the presence of concurrent accesses from multiple processes must be clearly defined and correctly implemented. The file system must have a mode that supports byte-level consistency; it could support additional modes with weaker consistency semantics. (By byte-level consistency we mean that if a process writes some number of bytes starting from some location in the file, the data written must be visible to other processes immediately after the write from this process returns, without requiring an explicit cache flush.) Unix or POSIX consistency semantics, which support byte-level consistency, are sufficient for implementing MPI-IO.

3. Atomicity Semantics. File systems can deliver higher performance if they are not required to guarantee atomicity of accesses. Furthermore, most applications do not perform concurrent overlapping accesses and, consequently, do not need the stricter atomic mode. We therefore recommend that the file system support two modes: an atomic mode and a higher-performance nonatomic mode. Some file systems, such as IBM PIOFS and Intel PFS, already support both modes.

\footnotetext{
${ }^{4}$ We thank Takao Hatazaki of HP-Convex for suggesting that we add this useful feature to ROMIO.
} 
4. File-Attribute Consistency. The file system must also support consistency of file attributes, such as file size. For example, if two processes open a new (nonexisting) file, one process writes 100 bytes to the file, and the other process then calls a function that returns the size of the file, the function must return the file size as 100 bytes. We encountered problems with this feature on NFS, because NFS caches file attributes on each process noncoherently. As a result, the second process read the file size as zero bytes. We solved this problem by mounting the NFS directory with the "noac" option (no attribute caching).

5. Interface Supporting Noncontiguous Accesses. Although an MPI-IO implementation can perform data sieving to access noncontiguous data with high performance, we believe that the performance can be even better if data sieving is done within the file system. (Note that when data sieving is done within the file system, it is no different from regular caching; the extra data read/written can remain in the cache and need not be discarded.) For this purpose, the file system must provide an interface that supports noncontiguous accesses. A simple interface in which the user specifies a list of offsets and lengths is sufficient. (See Section 3.2 for reasons why POSIX lio_listio is not appropriate.) A simple interface, such as the following, is desired:

int read_list(int mem_list_count, long long *mem_offsets, int *mem_lengths, int file_list_count, long long $*$ file_offsets, int $\left.* f i l e_{-} l e n g t h s\right)$

(similarly for write_list)

where mem_offsets and mem_lengths are lists of offsets and lengths representing noncontiguous memory locations, mem_list_count is the number of entries in mem_offsets and mem_lengths, file_offsets and file_lengths are lists of offsets and lengths representing noncontiguous locations in the file, and file_list_count is the number of entries in file_offsets and file_lengths. This interface can be considered as a generalization of Unix readv/writev to allow noncontiguity in the file.

In MPI-IO, noncontiguous data access with a single I/O function is allowed only to monotonically nondecreasing offsets in the file; memory offsets can be in any order. The read_list/write_list functions, therefore, need only allow monotonically nondecreasing offsets in file_offsets. This restriction can simplify the implementation of these functions.

6. Support Files Larger than 2 Gbytes. An increasing number of applications need to access files larger than 2 Gbytes. It is therefore critical that the file system be able to support large files. This means that the file-system interface and internal data structures must use 64-bit integers to represent file offsets.

7. Byte-Range Locking. The file system must support a locking facility equivalent to the advisory record-locking feature ( $f \mathrm{cntl}$ locks) in Unix and POSIX. ROMIO uses this feature to implement MPI-IO's atomicity semantics for noncontiguous file accesses, to implement the optimization data sieving for write requests, and to implement shared file pointers.

8. Control over File Striping. Since the best values for file-striping parameters often depend on the application's access pattern, we recommend that the file system use a "good" set of values as the default and provide a facility for users to vary these parameters on a per-file basis.

9. Variable Caching/Prefetching Policies. Parallel applications exhibit such a wide variation in access patterns that any one caching/prefetching policy is unlikely to perform well for all applications [26]. The file system must therefore either detect and automatically adapt to changing access patterns $[15,16]$ or provide an interface for the user to specify the access pattern or caching/prefetching policy $[2,21]$. 
10. File Preallocation. It is easy and inexpensive for a file system to provide a function to preallocate disk space for a file. If such a function is not provided, the MPI-IO function MPI_File_preallocate can be implemented only by actually writing data to the file, which is very expensive.

11. Leave Collective I/O to the MPI-IO Implementation. It is not entirely clear whether collective $\mathrm{I} / \mathrm{O}$ is better if performed in the file system or as a library above the file system. Both techniques have been proposed in the literature $[5,13,24]$. Our opinion is that collective I/O is a fairly complex optimization that, if done within the file system, could complicate the file system considerably. A file system must be able to deliver high performance to multiple simultaneous users and jobs and, therefore, must be as lightweight as possible. We have demonstrated in ROMIO that collective I/O can be effectively implemented on top of the file system and can deliver good performance [32]. We therefore believe that until parallel file systems mature sufficiently, it is better to leave collective I/O to the MPI-IO implementation.

12. No shared file pointers. We believe that the file system should not be burdened with the additional load of supporting shared file pointers. They can be implemented on top of the file system, as described in Section 3.8 .

13. Nonblocking (Asynchronous) I/O Optional. It is not mandatory for the file system to provide nonblocking I/O functions. An MPI-IO implementation can perform nonblocking I/O by using threads that call the blocking I/O functions. This method, however, requires proper thread support from the machine and a thread-safe MPI implementation.

We note that the semantics and interface provided by a POSIX file system are sufficient for implementing MPI-IO correctly (as ROMIO demonstrates), but additional features would help an MPI-IO implementation achieve higher performance. (ROMIO compensates for the absence of these features by performing optimizations such as data sieving and collective I/O.) Of the features listed above, those not supported in POSIX are an interface for noncontiguous accesses, control over file striping, hints for caching/prefetching policies, and file preallocation. High-performance parallel file access and file sizes larger than 2 Gbytes are not mandated by POSIX but are considered "implementation-dependent features."

\section{Conclusions}

ROMIO demonstrates that it is possible to implement MPI-IO portably on multiple machines and file systems and also achieve high performance. The ADIO framework is the key component that makes this all possible, as it enables us to perform file-system-specific optimizations within a largely portable implementation.

The discussion in this paper covers numerous file systems-almost all the file systems on commercially available machines. An important storage system that we did not discuss (mainly because ROMIO is not implemented on it) is HPSS [36]. HPSS is different from other file systems in its goals and design features; for example, it supports third-party transfer. A group at Lawrence Livermore National Laboratory has implemented MPI-IO on HPSS, and we refer interested readers to [12] for a discussion of issues related to implementing MPI-IO on HPSS.

By making MPI-IO available everywhere and also delivering high performance, we expect it will be widely used and popular among application programmers. We believe it will solve some of the I/O performance and portability problems currently experienced in parallel applications. 


\section{References}

[1] S. Baylor and C. Wu. Parallel I/O Workload Characteristics Using Vesta. In R. Jain, J. Werth, and J. Browne, editors, Input/Output in Parallel and Distributed Computer Systems, chapter 7, pages 167-185. Kluwer Academic Publishers, 1996.

[2] P. Cao, E. Felten, A. Karlin, and K. Li. Implementation and Performance of Integrated ApplicationControlled File Caching, Prefetching, and Disk Scheduling. ACM Transactions on Computer Systems, 14(4):311-343, November 1996.

[3] P. Corbett, J. Prost, C. Demetriou, G. Gibson, E. Reidel, J. Zelenka, Y. Chen, E. Felten, K. Li, J. Hartman, L. Peterson, B. Bershad, A. Wolman, and R. Aydt. Proposal for a Common Parallel File System Programming Interface, Version 1.0. On the World-Wide Web at http://www.cs.arizona.edu/sio/api1.0.ps.gz, September 1996.

[4] P. Crandall, R. Aydt, A. Chien, and D. Reed. Input-Output Characteristics of Scalable Parallel Applications. In Proceedings of Supercomputing '95. ACM Press, December 1995.

[5] J. del Rosario, R. Bordawekar, and A. Choudhary. Improved Parallel I/O via a Two-Phase Runtime Access Strategy. In Proceedings of the Workshop on I/O in Parallel Computer Systems at IPPS '93, pages 56-70, April 1993. Also published in Computer Architecture News, 21(5):31-38, December 1993.

[6] S. Fineberg, P. Wong, B. Nitzberg, and C. Kuszmaul. PMPIO-A Portable Implementation of MPIIO. In Proceedings of the Sixth Symposium on the Frontiers of Massively Parallel Computation, pages 188-195. IEEE Computer Society Press, October 1996.

[7] I. Foster, D. Kohr, R. Krishnaiyer, and J. Mogill. Remote I/O: Fast Access to Distant Storage. In Proceedings of the Fifth Workshop on Input/Output in Parallel and Distributed Systems, pages 14-25. ACM Press, November 1997.

[8] G. Gibson, D. Stodolsky, P. Chang, W. Courtwright II, C. Demetriou, E. Ginting, M. Holland, Q. Ma, L. Neal, R. Patterson, J. Su, R. Youssef, and J. Zelenka. The Scotch Parallel Storage Systems. In Proceedings of 40th IEEE Computer Society International Conference (COMPCON 95), pages 403-410. IEEE Computer Society Press, Spring 1995.

[9] W. Gropp, E. Lusk, N. Doss, and A. Skjellum. A High-Performance, Portable Implementation of the MPI Message-Passing Interface Standard. Parallel Computing, 22(6):789-828, September 1996.

[10] J. Huber, C. Elford, D. Reed, A. Chien, and D. Blumenthal. PPFS: A High Performance Portable Parallel File System. In Proceedings of the 9th ACM International Conference on Supercomputing, pages 385-394. ACM Press, July 1995.

[11] IEEE/ANSI Std. 1003.1. Portable Operating System Interface (POSIX)-Part 1: System Application Program Interface (API) [C Language], 1996 edition.

[12] T. Jones, R. Mark, J. Martin, J. May, E. Pierce, and L. Stanberry. An MPI-IO interface to HPSS. In Proceedings of the Fifth NASA Goddard Conference on Mass Storage Systems, pages I:37-50, September 1996.

[13] D. Kotz. Disk-directed I/O for MIMD Multiprocessors. ACM Transactions on Computer Systems, 15(1):41-74, February 1997. 
[14] O. Krieger and M. Stumm. HFS: A Performance-Oriented Flexible File System Based on BuildingBlock Compositions. In Proceedings of Fourth Workshop on Input/Output in Parallel and Distributed Systems, pages 95-108. ACM Press, May 1996.

[15] T. Madhyastha and D. Reed. Intelligent, Adaptive File System Policy Selection. In Proceedings of the Sixth Symposium on the Frontiers of Massively Parallel Computation, pages 172-179. IEEE Computer Society Press, October 1996.

[16] T. Madhyastha and D. Reed. Exploiting Global Input/Output Access Pattern Classification. In Proceedings of SC97: High Performance Networking and Computing. ACM Press, November 1997.

[17] Message Passing Interface Forum. MPI-2: Extensions to the Message-Passing Interface. July 1997. On the World-Wide Web at http://ww .mpi-forum.org/docs/docs.html.

[18] Message Passing Interface Forum. MPI: A Message-Passing Interface Standard. Version 1.1, June 1995. On the World-Wide Web at http://ww .mpi-forum.org/docs/docs.html.

[19] N. Nieuwejaar and D. Kotz. The Galley Parallel File System. Parallel Computing, 23(4):447-476, June 1997.

[20] N. Nieuwejaar, D. Kotz, A. Purakayastha, C. Ellis, and M. Best. File-Access Characteristics of Parallel Scientific Workloads. IEEE Transactions on Parallel and Distributed Systems, 7(10):10751089, October 1996.

[21] R. Patterson, G. Gibson, E. Ginting, D. Stodolsky, and J. Zelenka. Informed Prefetching and Caching. In Proceedings of the 15th Symposium on Operating System Principles, pages 79-95. ACM Press, December 1995.

[22] J. Prost. MPI-IO/PIOFS. World-Wide Web page at http://www.research.ibm.com/people/p/prost/sections/mpiio.html, 1996.

[23] D. Sanders, Y. Park, and M. Brodowicz. Implementation and Performance of MPI-IO File Access Using MPI Datatypes. Technical Report UH-CS-96-12, University of Houston, November 1996.

[24] K. Seamons, Y. Chen, P. Jones, J. Jozwiak, and M. Winslett. Server-Directed Collective I/O in Panda. In Proceedings of Supercomputing '95. ACM Press, December 1995.

[25] E. Smirni, R. Aydt, A. Chien, and D. Reed. I/O Requirements of Scientific Applications: An Evolutionary View. In Proceedings of the Fifth IEEE International Symposium on High Performance Distributed Computing, pages 49-59. IEEE Computer Society Press, 1996.

[26] E. Smirni and D. Reed. Lessons from Characterizing the Input/Output Behavior of Parallel Scientific Applications. Performance Evaluation: An International Journal, 33(1):27-44, June 1998.

[27] H. Stern. Managing NFS and NIS. O’Reilly \& Associates, Inc., 1991.

[28] W. Stevens. Advanced Programming in the UNIX Environment. Addison-Wesley Publishing Company, Inc., 1992.

[29] R. Thakur and A. Choudhary. An Extended Two-Phase Method for Accessing Sections of Out-ofCore Arrays. Scientific Programming, 5(4):301-317, Winter 1996.

[30] R. Thakur, W. Gropp, and E. Lusk. An Abstract-Device Interface for Implementing Portable Parallel-I/O Interfaces. In Proceedings of the 6th Symposium on the Frontiers of Massively Parallel Computation, pages 180-187. IEEE Computer Society Press, October 1996. 
[31] R. Thakur, W. Gropp, and E. Lusk. An Experimental Evaluation of the Parallel I/O Systems of the IBM SP and Intel Paragon Using a Production Application. In Proceedings of the 3rd International Conference of the Austrian Center for Parallel Computation (ACPC) with Special Emphasis on Parallel Databases and Parallel I/O, pages 24-35. Lecture Notes in Computer Science 1127. Springer-Verlag, September 1996.

[32] R. Thakur, W. Gropp, and E. Lusk. Data Sieving and Collective I/O in ROMIO. Technical Report ANL/MCS-P723-0898, Mathematics and Computer Science Division, Argonne National Laboratory, August 1998.

[33] R. Thakur, E. Lusk, and W. Gropp. Users Guide for ROMIO: A High-Performance, Portable MPIIO Implementation. Technical Report ANL/MCS-TM-234, Mathematics and Computer Science Division, Argonne National Laboratory, October 1997.

[34] R. Thakur, E. Lusk, and W. Gropp. I/O in Parallel Applications: The Weakest Link. International Journal of High Performance Computing Applications, 1998. To appear.

[35] The MPI-IO Committee. MPI-IO: A Parallel File I/O Interface for MPI, Version 0.5. On the World-Wide Web at http://parallel.nas.nasa.gov/MPI-I0, April 1996.

[36] R. Watson and R. Coyne. The Parallel I/O Architecture of the High-Performance Storage System (HPSS). In Proceedings of the Fourteenth IEEE Symposium on Mass Storage Systems, pages 27-44. IEEE Computer Society Press, September 1995. 